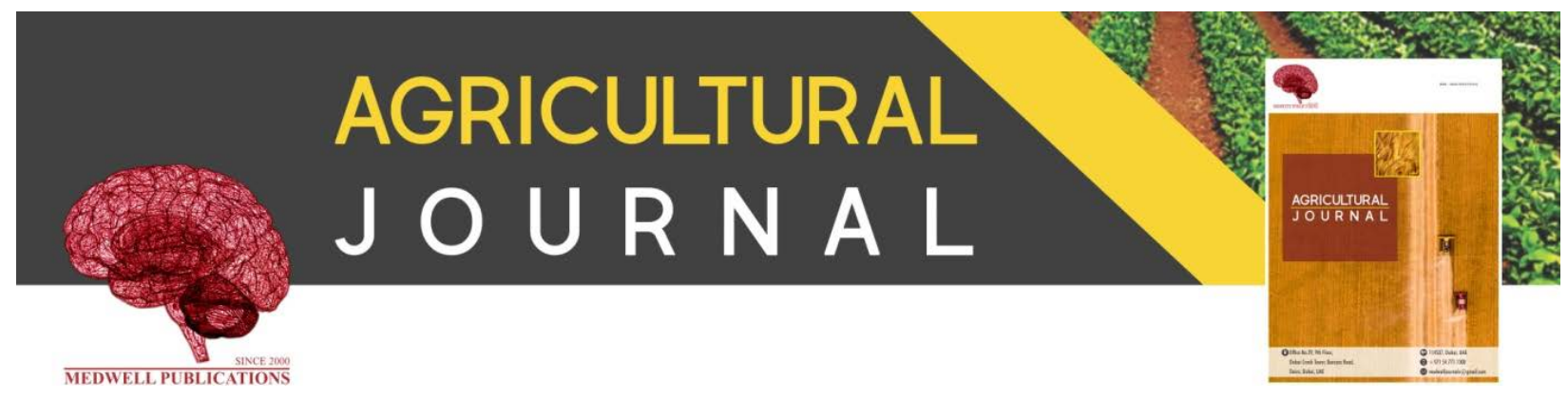

\title{
IoT Based Water Monitoring System for Agriculture
}

Heena Nankani, Shruti Gupta, Shubhadeep Mondal and S. Kalaiarasi SRM Institute of Science and Technology, Ramapuram, 600089 Chennai, India

Key words: Flow sensor, Arduino, WI-FI system, cloud server, safety

\section{Corresponding Author:}

Heena Nankani

SRM Institute of Science and Technology, Ramapuram, 600089 Chennai, India

Page No.: 37-41

Volume: 15, Issue 3, 2020

ISSN: 1816-9155

Agricultural Journal

Copy Right: Medwell Publications
Abstract: Water being the utmost need for everyone in today's world needs to be pure and safe for everyone. This study presents a model on smart water monitoring which holds quantity and quality of water for agriculture, irrigation or drinking purposes. Thus, a model containing Arduino and some sensors for detection of contamination, turbidity, temperature, water level and water flow. The micro-controller Arduino is connected with the sensors which collect the data and then displays the data on the screen. The WI-FI module is used to make a connection with the Arduino which collect all the datas which are sensed by the sensors and then is stored in the cloud server. This model is highly useful for the future safety of the water for the next generation.

\section{INTRODUCTION}

From ancient time, agriculture is performed in almost every country. Agriculture is the art and scientific logic for cultivation. Agriculture is the key to develop every country. Agriculture is done manually and planned well. In the present situation many new technologies have played a vital role in the wide process of development, so, agriculture needs to cooperate with those new technologies. IoT has a very important role in smart agriculture. IoT smartly records every possible thing required for the quality and quantity monitoring in agriculture fields with the help of sensors. IoT based smart monitoring have been proposed by us. The sensors will sense all the data or the values which are will be monitored while the sensors will work along with the Arduino and the values will be transferred with the help of a wireless network or the WI-FI-module. This smart water monitoring system is powered by Arduino, sensors names water level sensor, water flow sensor, IR sensor, turbidity sensor, $\mathrm{PH}$ sensor and a WiFi module to store the data in the cloud. When the monitoring of the water starts it checks the water level, water flow, $\mathrm{pH}$ of the water, contamination of the water, temperature and turbidity of the water. Connection is done in such a manner that a notification or a SMS is send to respective connected android phones to know about the water levels so that the tap can be closed on time. If the level of water goes down, the water level sensor sense the level of water and automatically starts the water pump The temperature sensors sense the temperature of the water and starts the fan when the temperature goes high. This all is displayed on the LCD display module. The $\mathrm{pH}$ value and the contamination is also displayed on the screen.. Temperature can be set on a particular level, it is based on the type crops cultivated.

Water safety is utmost important because it is used in agriculture, consumption and travel, thus, it is important to keep on monitoring the quality and quantity of water. Water thus, contains chemical impurities like redox, dissolved oxygen, ammonium on the surface bodies causes water pollution and thus, $90 \%$ of the river basin management indicated agriculture to be most pressurised in the basin. Thus, this plan is to reduce the nitrogen and potassium loads in the water surface bodies and those agricultural waste that are running in the water bodies 
from the urban areas. Therefore, the time has come to improve the monitoring system for the good quality and the proper quantity of water, so, to increase the public healthcare and reducing the problems they are facing. Improved planning is done for the welfare of the economy and our environment for the future purposes.

Literature survey: In recent years, few works have been reported in the literature for the smart water monitoring system and about measuring the quality and quantity of water using different sensors by the mechanism of IoT.

Various reports have been created and much hard work has already been performed for implementing the water monitoring model.

Liu et al. (2018) says that the results of water quality monitoring performed by the Daheiting Reservoir. The initial or introductory investigation of the reservoir for water quality mechanism is mentioned in the following paragraph. It is observed from the analyzed data that the reservoir is affected by the dynamic conditions of water, meteorological and scheduling conditions. The water body being present in the reservoir area has seasonal temperature stratification. The temperature of water stratification ranges from April to May every year.

Manoharan and Rathinasabapathy (2018) presented a paper in which they told about a survey done on the recent researches in smart villages. The distribution and monitoring of the quality of water in areas such as villages as well as cities is a stimulating piece of work. The solution for smart water distribution as well as quality monitoring in villages is primarily discussed and the solution is provided using LoRa and LoRaWAN. LoRaWAN provides an alternate solution for networking where the cellular network is not available. This LoRaWAN is suitable in forest areas and mountains where the cellular network is not available as well in cities where the cellular network couldn't provide service to these types of applications. The proposed system will save water and provide good quality of water to people.

Kulkarni and Farnham (2016) his article provided an overview of issues involved in water monitoring and management and highlighted the wire-less connectivity considerations to enable smart water man-agement. Challenges and design considerations involved in choosing from a variety of architectural approaches to realise communications were highlighted. While there is no doubt that communications heavily underpins the successful deployment of a water management strategy, the choice of a specific solution boils down to the objectives and constraints of the scenario in question. A TCO based framework was proposed which can assist stakeholders for whom communications may not be a core business area, to assess the cost of the different solutions and the potential implications of choices that are made. The application of this framework was also demonstrated as a part of the ICeWater project through smart water case studies in the water networks of two utilities in Europe.

Saab et al. (2017) presented the paper in which they analyzed the use of two smart sensors namely: S:: CAN and EventLab. These two smart sensors are used for the early detection of water contamination. Pilot station was used in the beginning for checking the performance. Followed by this, the sensors were put in the distributed network of the Scientific Campus of the University of Lille. Registered data represented the quasi-constant signals. A comparing act was performed between registered data and laboratory analyzed data which ensured the good performances of the tested sensors.

\section{MATERIALS AND METHODS}

Figure 1 determines the architecture diagram of the smart water monitoring system for agriculture. Thus, the sensors are used for monitoring quality and quantity of the water. The ph detector, Turbidity detector, Temperature sensor also the flow sensor, water level detector. These detectors are used to monitor quality and quantity of water. The values which are obtained from the detectors are accessed by the Arduino device and thus, all thee database are stored in the cloud with the help of the WiFi-module, so, the data's can be used in the future purpose for the data analytics.

\section{Architecture diagram}

pH sensor: The $\mathrm{pH}$ is used to measure the water quality. It is basically a measurement of acidity and alkalinity. It ranges from $0-14$. The $\mathrm{pH}$ scale of acidic ranges from 0-7 and the range of alkalinity varies from 8-14. The value 7 represents the neutral state of the water.

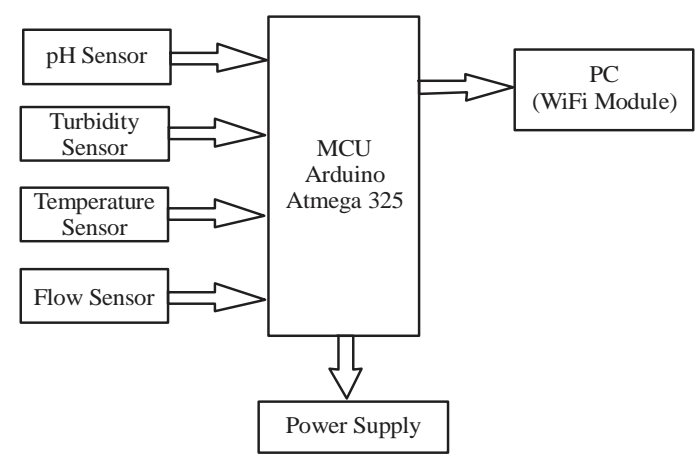

Fig. 1: Architecture diagram 
Agric. J., 15 (3): 37-41, 2020

Out of all the most used technique to measure the water quality is the ph sensor which is the electrochemical based. These are the kind of the ph detectors that has both the things. A electrode which is used for measuring and a electrode for reference. The electrode which is used for measuring determines the $\mathrm{pH}$ fluctuation whereas the data gives signals to compare. Electrochemical $\mathrm{pH}$ detector can be used to make various products such as process $\mathrm{pH}$ detectors. Some of the applications of the electrochemical $\mathrm{pH}$ sensors are:

- $\mathrm{pH}$ monitoring

- $\quad$ Pre-treatment of the water

- Manufacturing of pulp and paper

- Quality control and safety such as food and beverage quality

Turbidity sensor: It is required to check and determine the status of the quality of the water. Water may have floating materials in it which consists of the materials that are having different varying dimensions. While some floating material are large also heavy enough to get settled in the bottom of the tank if a liquid is left to get settled, very minute particles will settle. This process is very slowly or not at all if the particles are opaque. Turbidity can also be applied to non opaque solids.

Water alone develops some of the rigidity and any particle which is set free in the water that develops color may develops a wavelengths. The intensity of particles to spread a light focused on them is now said to be as a more meaningful measuring activity of the requirement in the water. Turbidity measured in this way uses a device which is known as nephelometer. Intense amount of light will resist to the detecting machine if there are many small substances spreading the source ray than the some. Nephelometric Turbidity Units is also known as the units of turbidity.

Turbidity at the water bodies can be mapped using a disk called as Secchi disk. The disk has the plus point of turbidity over the amount of depth being simple and easy to use and inexpensive. An additional device which is called as turbidity tube may be needed in mapping the turbidity in deep water bodies.

Temperature sensor: Matter which is formed by the movement of the particles, every of which is having its own developing speed and also the energy. The flash point is also a physical point which describes the approx energy of the substances, it isn't not only a criteria of the energy only but it is directly dependent to the approx energy of substances. It means the hot molecules are, rapid in their movement and the higher in the flsh points. Whenever, the particles stay in constant or static motion. A device known as thermometer is an instrument required for the measurement of the flash points.
Flow sensor: A flow detector is a measuring system that is required to detect the flow rate or q liquid passing through a pipeline, for example, a water pipeline. Flow sensors are also known by other names, namely as flow gauge, liquid meter, etc. Which depends on the particular industry. The flow is also represented by Q. Q is same as the cross-sectional area of pipe which is represented by $\mathrm{A}$ in a meter and the distanve per unit time of the flowing and passing of the liquid.

Water level sensor: This sensor detects the level of water in the water bodies mainly reservoir and the tanks which mainly consists of the water tanks. This sensor mainly works in analyzing the leakage of the water as well as water level. This sensor is formed with three major parts in it. A resistor used for the resistance, an electronic brick which can be used as a connector and various lines of the conducting wires. A weak resistor of $1 \mathrm{MO}$ is present This can test the fluid drop by using a bunch of the parallel wires.

IR sensor: Observing and verifying the water quality with a attenuing focus on organic harmful substances is priority of the entire world topic in the feild of analyzation of the water. Sensing things in the infrared are among very helping techniques due to its functioning in the specific provided by the pattern of the absoarbtion of the most basic molecular vibrations of organic substances. It is an electronic device. This is used to know the things from it near about. It does things by the emission of this or either by detecting the emissions which are caused by the infrared. These are also able to measure the heat energy which is being emitted by any material and detecting motion.

Arduino: Arduino is open software which is also hardware that designs and makes uni-board kits used for developing digital devices. Its products are licensed under GNU or GNU which permits the making process of the Arduino boards and software division by someone. These devices are available in the commercial areas and are readily assembled by the manufacturer.

The designs of the device use a variety of small other devices such as microprocessors or the microcontrollers. The devices are plugged with the sets of the digital pins which are used for the connection the circuits. The boards are programmed using $\mathrm{C}$ and $\mathrm{C}++$ programming languages which are pre-defined for the Arduino software.

This project of Arduino started in the year 2005 as a program for students. Some of the common examples of such devices include simple robots and motion detectors and many more. Figure 2 represents the Arduino. 


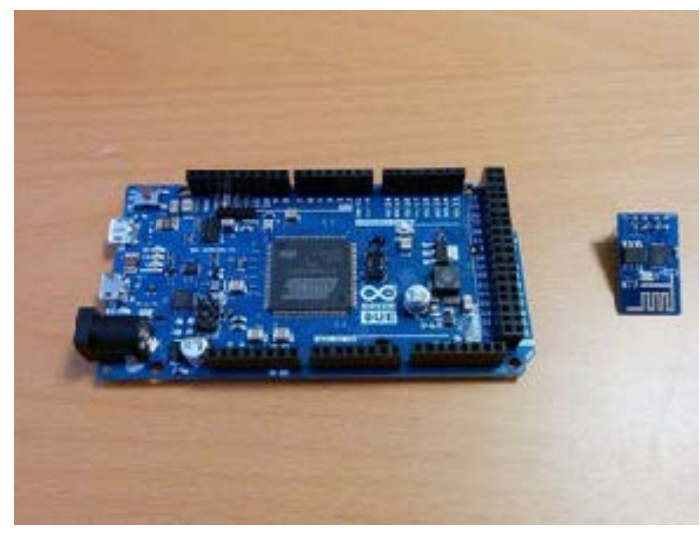

Fig. 2: Arduino

WiFi-module: The Wi-Fi module used is the ESP8266.TCP stack is used in its mechanism and it is having a microchip. This microchip allows to connect to the internet. It is a low-cost compact chip which is having the complete TCP architechture also its capabilities.

The microchip first time came to the scenario of the Western makers. The compact module helps us to connect to the internet and make simple TCP connections. In this module ESP8285, an ESP8266 with the help of the architecture of built-in flash which allows the building of single chip device able of connect to the internet and with the other devices inn the network.

\section{RESULTS AND DISCUSSION}

Experimental results and discussion: We tested the prototype system and we got the following experimental results according to our study.

Flow sensor: When we are passing the water from the flow sensor, the programmed Arduino is connected to the flow sensor. This allows the sensor to measure the current flow rate of the water and experimental data of the same gets stored in the cloud database which we can access from any device we want. Figure 3 represents the working of the workflow sensor.

pH sensor: When we are passing the water from the ph sensor, the programmed Arduino is connected with the ph sensor. This allows you to measure the $\mathrm{pH}$ level of the water. The output will be more if the $\mathrm{pH}$ level is very high and the output will be very less if the $\mathrm{pH}$ value will be very low. With this, we can understand the acidity and the basic nature of the water. The resulting output will be shown in a cloud-based database which can be accessed from any device.

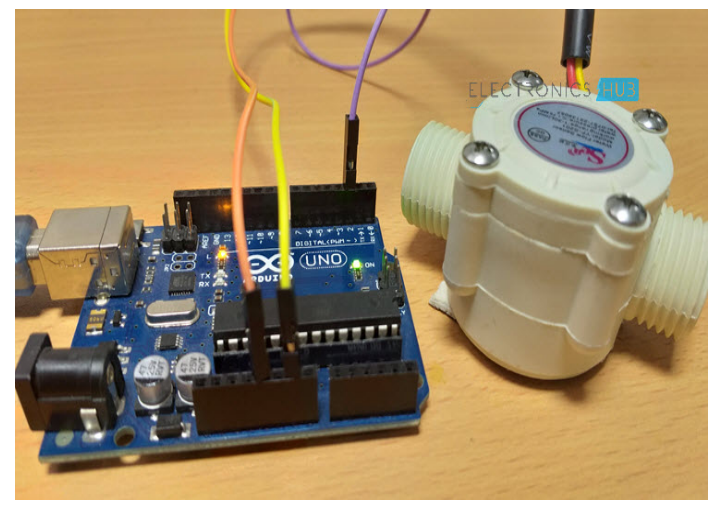

Fig. 3: Water flow sensor

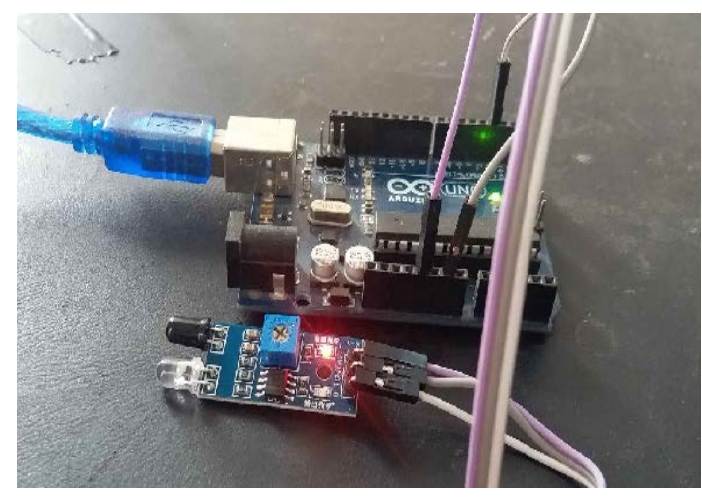

Fig. 4: IR sensor

IR sensor: When we are passing the water from the IR sensor, the programmed Arduino is connected with the IR sensor. This IR sensor allows us to detect any harmful solid wastes in the water. When the water will pass from the IR sensor in the pipe the harmful solid wastes will be detected and a buzzer with light will be shown to the user. In the presence of solid wastes, the sensor will give " 1 " as the output or else " 0 " will be the output. The result will be stored as an output in a cloud-based database and can be assessed from any device. Figure 4 represents the working of the IR sensor.

Turbidity sensor: When we are passing the water from the turbidity sensor, the programmed Arduino is connected to the turbidity sensor. The turbidity sensor will emit a beam of light in the water to check the quality of the water. If the water is opaque or thick then it will beep an alarm and the sensor will not give " 000 " as output. If the water is clear then the output will be " 000 ". The output will be recorded in a cloud database.

Temperature sensor: When we are passing the water from the temperature sensor, the programmed Arduino is 


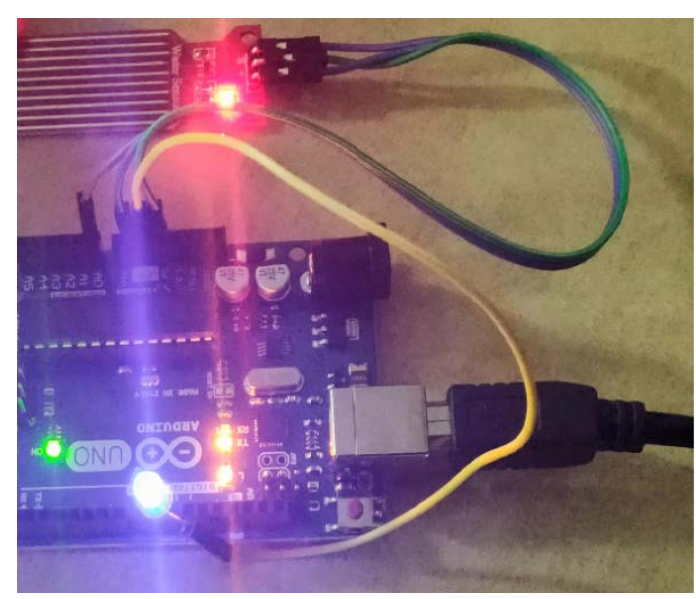

Fig. 5: Water level sensor

connected to the temperature sensor. The kinetic energy of the moving particles will increase the temperature of the water which is not suitable for agriculture. If the temperature is more than the required, then it will show "WRONG" as an output. If correct, then it will show "OK" as an output. The result will be recorded in a cloud bases database and can be accessed from anywhere.

Water level sensor: When the water level of the tank is preceding the specified amount of water level then a buzzer along with a light is being shown by the sensor. The sensor is programmed using a Arduino board. After getting the observed output, it will be stored in the cloud database using the ESP $8266 \mathrm{WiFi}$ module and can be accessed from any device later. Figure 5 represents the working of the water level sensor. Hence, the prototype system is working correctly will a accuracy level of $95 \%$.

\section{CONCLUSION}

The contribution of our study is to prove the feasibility and effectiveness of the monitoring of water which is being supplied for agriculture. Thus the water supplied should be perfect in ph, temperature and quantity for proper yielding of crops. Thus, IoT sensors helped to create a successful water monitoring model for detecting $\mathrm{ph}$ of the water, temperature of the water flowing which determines the quality of the water and determining the flow of water using flow sensor and the water level sensor for determining the level of water flowing. This projects helped out in agriculture field.

\section{REFERENCES}

Kulkarni, P. and T. Farnham, 2016. Smart city wireless connectivity considerations and cost analysis: Lessons learnt from smart water case studies. IEEE. Access, 4: 660-672.

Liu, C., L. Wang, B. Li, S. Wang and Y. Du, 2018. Analysis of water temperature stratification and water quality response mechanism of daheiting reservoir in Tangshan City. Proceedings of the 2018 3rd International Conference on Smart City and Systems Engineering (ICSCSE), December 29-30, 2018, IEEE, Xiamen, China, pp: 849-854.

Manoharan, A.M. and V. Rathinasabapathy, 2018. Smart water quality Monitoring and metering using Lora for smart villages. Proceedings of the 2018 2nd International Conference on Smart Grid and Smart Cities (ICSGSC), August 12-14, 2018, IEEE, Kuala Lumpur, Malaysia, pp: 57-61.

Saab, C., I. Shahrour and F.H. Chehade, 2017. Smart technology for water quality control: Feedback about use of water quality sensors. Proceedings of the 2017 Sensors Networks Smart and Emerging Technologies (SENSET), September 12-14, 2017, IEEE, Beirut, Lebanon, pp: 1-4. 